\title{
The dark perimetric stimulus
}

\author{
Erkan Mutlukan, Bertil E Damato
}

\begin{abstract}
We determined the disappearance eccentricities of dark and bright stimuli of equal size in the inferonasal central visual field using the oculokinetic perimetry technique at different levels of surrounding illumination. The results suggest that a dark stimulus on a bright background has a smaller 'isoptre' than an equally bright stimulus on a dark background, and that variation of ambient illumination and consequent alteration of background luminance have less effect on the visibility of a dark stimulus than a bright one.
\end{abstract}

The visual field is conventionally examined with a light stimulus on a dark background. This choice of perimetric stimulus probably stems from the assumption that the fundamental role of the retina is to detect light. ${ }^{1-3}$ However, the retina is not merely a light sensor but is primarily adapted for the detection of differences, both increments and decrements, in the amount of light in the visual field. The functional organisation and grouping in ganglion cell receptive fields in the retina provides evidence for this. Stimulation occurs only if there is a difference in the amount of light falling on the receptive field centre and on the surrounding area. ${ }^{4-9}$ One group of the receptive fields is activated by light falling into the field centre and is therefore called the 'on' type. Conversely, the other group, namely the 'off' type receptive fields, activate the ganglion cell with the cessation (decrement) of the light.

It is believed that the visibility of a perimetric stimulus is related to the number of overlapping receptive fields in the corresponding part of the retina, which is in turn related to the density of the ganglion cells in that region. ${ }^{10-13}$ It is now known that nerve fibres from the retinal ganglion cells travel to the visual cortex in two parallel visual pathways, namely 'on' and 'off' systems which remain morphologically, physiologically, and pharmacologically separate until they converge on single cortical neurones. ${ }^{12} 1420$

In the $25^{\circ}$ central visual field most ganglion cell receptive fields $(60-75 \%)$ have 'on centre' organisation, so that they are stimulated by a light spot on a dimmer background (that is, incremental light stimulus') and are inhibited by a central light decrement. Conversely, with 'off centre' ganglion cells, maximal stimulation occurs with a dark stimulus on a brighter background. ${ }^{7}{ }^{12} 1521$ Whereas conventional perimetry tests onpathway and mainly on-centre receptive fields at various locations across the retina, there may be advantages in testing the off-pathway by a dark stimulus because of the difference in the quantity of reserves in both systems, as it is possible that field defects that are missed with a conventional light stimulus might be detected earlier in certain conditions, such as glaucoma and intracranial disorders affecting the visual system.

The presentation of a dark stimulus on a white tangent screen is difficult, as it would require a white wand which would lead to confusion by casting a dark shadow. This technical difficulty is overcome by using the 'oculokinetic multifixation campimetry technique,' which does not require the stimulus to be moved on the screen, because it is the patient's eye which moves instead. ${ }^{22}$

The aim of our study was to identify differences between responses to dark and light perimetric stimuli in the central visual field by the oculokinetic technique and to determine how these responses vary with changes in ambient illumination.

\section{Material and methods}

The study involved the assessment of the visual fields of the right eyes of eight volunteers, six of whom were male and two female. Their visual acuities were all $6 / 5$, and the pupil sizes ranged between 3 and $5 \mathrm{~mm}$ in 150 lux room illumination. All eyes were normal.

The experiments were performed with three different types of tangent screen: black, white and grey, having a light reflectance (albedo) of $5 \%, 95 \%$, and $50 \%$ respectively. Each test stimulus consisted of a disc attached on to the screen which had a light reflectance of either $5 \%$ (black) or $95 \%$ (white), creating equal but opposite sign luminance (Weber's) contrast at a given illuminance level (Fig 1). The fixation target consisted of a pointer, which was moved across the screen superotemporally by the examiner, so that the pursuit movements of the subject's eye induced a relative shift of the stimulus along the $225^{\circ}$ meridian in the central visual field. The eccentricity from fixation at which the stimulus was no longer seen was recorded. This procedure was repeated five times for each target and illuminance level, and a mean value was taken as the final result.

The tangent screen was illuminated with a white light halogen lamp fitted with a diffuser and a dimmer switch which was positioned at 2 metres from the screen. The illumination of the screen was measured and adjusted with a luxmeter (Solex SL100). Every time the ambient illumination was altered, 3 minutes were allowed to elapse before the examination was begun so as to ensure the subject's retinal light adaptation had taken place. The subject was seated, with the head resting on a chin rest so that the right eye was $150 \mathrm{~cm}$ from the screen. The left eye was covered with an occluder. All perimetric examinations were performed by the same individual, and all tests for each subject were completed on the same day.

Meridional hill of vision to dark and bright

Tennent Institute of
Ophthalmology,
University of Glasgow,
38 Church Street,
Glasgow G11 6NT
E Mutlukan
B E Damato
Correspondence to:
Dr Erkan Mutlukan.
Accepted for publication
21 November 1991



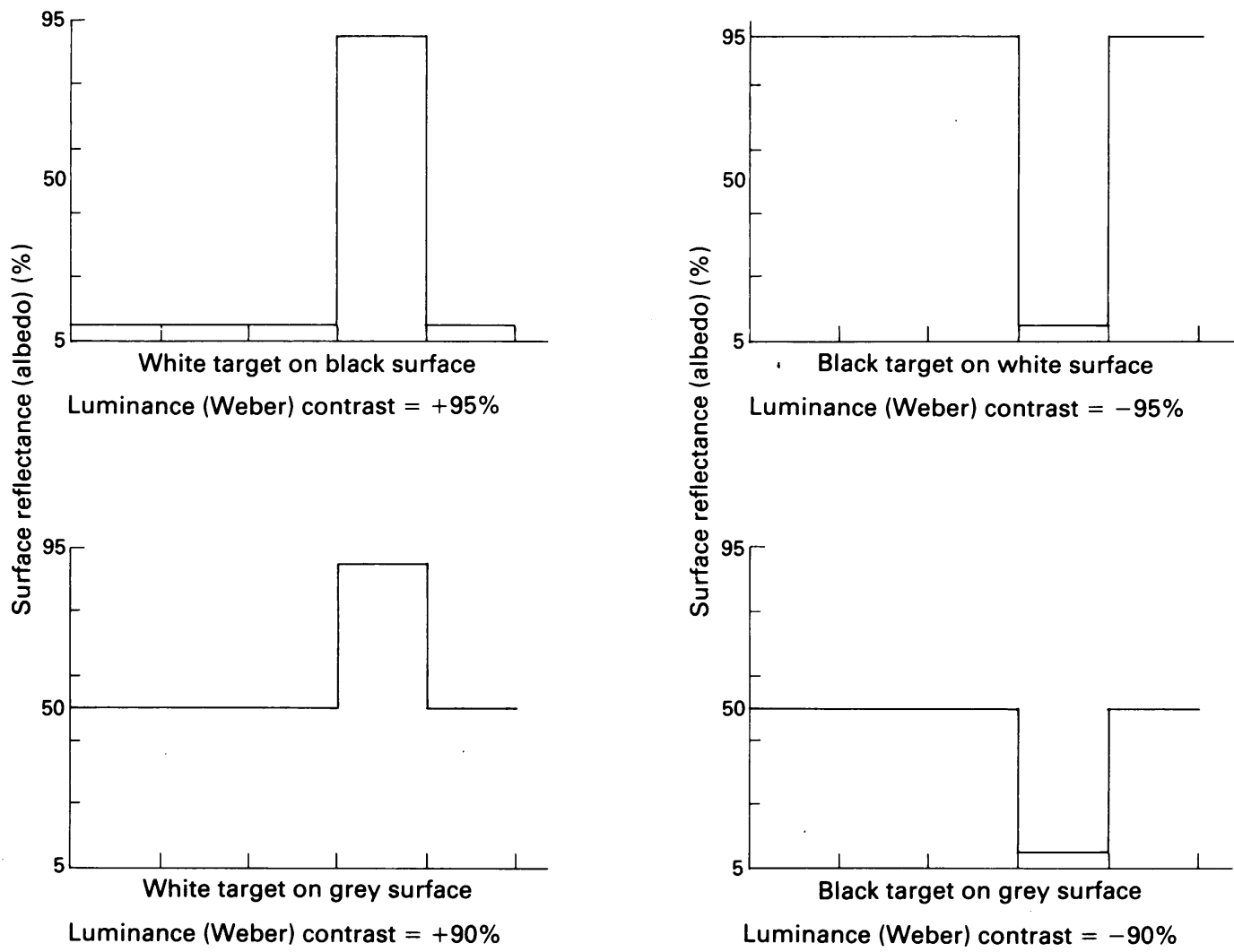

Figure 1 Luminance profiles and contrast values of four target-background combinations employed in the study. Luminance (Weber) contrast $=\left(L_{\text {Target }}-L_{\text {Background }}\right) / L_{\text {Backgroumd }}(L=$ luminance $)$.

stimuli. Four volunteers (two males and two females; mean age 30 years, range $28-31$ years) were tested with each of five black stimuli $(1,2$, 3 , 4, and $5 / 1500 \mathrm{~mm}$ ) against a white background, and also with white targets of the same size on a black background. The ambient illumination was 150 lux. The peripheral visibility of both stimulus types on the $225^{\circ}$ meridian was compared.

Effect of variation in illumination on dark and bright stimuli. The effect of variation in ambient illumination on the eccentric visibility of black and white stimuli on the $225^{\circ}$ meridian was examined at two levels of illumination, which were 13 and 400 lux (that is, a difference of 1.5 $\log$ units).

Eight volunteers (mean age 30 years, range 2539) were tested with each of the following four stimulus-background combinations in a random order: white-on-black, black-on-white, whiteon-grey, and black-on-grey. The grey background was employed in order to neutralise the difference in retinal light adaptation levels to different backgrounds, and to establish whether the results were due to the stimulus or the background. In each case a $1 / 1500 \mathrm{~mm}(2.5 \mathrm{~min})$ stimulus was employed. The percentage change in stimulus visibility with change in illumination was calculated with the formula:

$$
[(I-i) / i] \times 100 \text {, }
$$

where $I$ is the isoptre at 400 lux and $i$ is the isoptre at 13 lux. The percentage change in the isoptre of the black stimulus was compared with that of the white stimulus by the paired $t$ test.

\section{Results}

Comparison of visibility of black stimuli on white background and white stimuli on black background. The disappearance eccentricities of the black stimuli in the meridian tested were significantly smaller than those of the white stimuli, the difference being more pronounced with the smaller stimulus sizes (paired $t$ test, $\mathrm{p}<0.0001$ ) (Fig 2).

Effect of variation of illumination on black and white stimuli. The percentage increase in whiteon-black stimulus disappearance eccentricity was significantly more than that of black-onwhite stimulus $(t$ test, $\mathrm{p}=0.014)$. Increasing the ambient illumination from 13 lux to 400 lux increased the isoptre of the white stimulus by $59 \%$ and the isoptre of black stimulus by $36 \%$ (Fig 3a).

Similarly, the eccentric visibility change due to illuminance variation was significantly more for the white-on-grey stimulus than the black-ongrey stimulus ( $t$ test, $\mathrm{p}=0.0016$ ). At the higher ambient illumination, the black and white stimuli disappearance eccentricities increased by $75 \%$ and $117 \%$ respectively (Fig $3 b$ ). Isoptre increases of lower contrast black-on-grey stimulus and white-on-grey stimulus were significantly more pronounced than those of black-on-white ( $t$ test, $\mathrm{p}<0.0001)$ and white-on-black stimuli ( $t$ test, $\mathrm{p}=0.0069$ ).

\section{Discussion}

The campimetry was performed by the oculokinetic technique so as to be able to use a fixed 


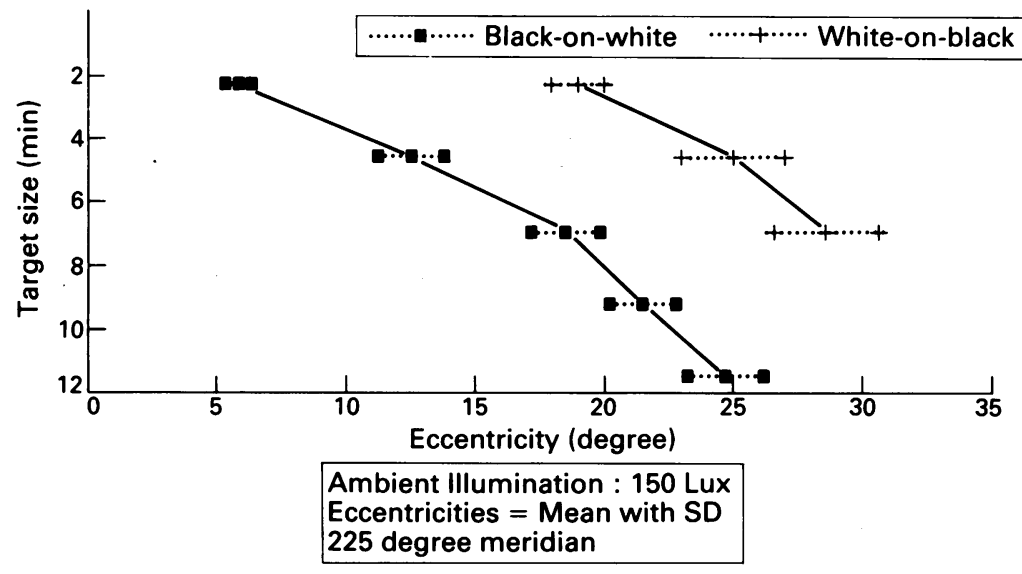

Figure 2 Peripheral visibility of various sizes of equal dark and bright stimuli in the inferonasal visual field (meridional hill of vision). The dark stimuli are significantly less visible than the bright stimuli of equivalent size.

test stimulus, thereby eliminating the need for manually moving the stimulus using a wand, which would have caused a shadow on the background, and which could also have resulted in tilting of the stimulus. A moving fixation target was used instead of several printed numbers on the screen, as previously described, so as to prevent bias caused by the patient memorising the numbers associated with disappearance of the stimulus. ${ }^{22}$ Contrary to routine conventional practice, the stimulus moved centrifugally. Although it is well known that isoptres are different when the direction of stimulus movement is from seen-to-unseen compared with unseen-to-seen, pilot studies had indicated that, when the percentage change in isoptre eccentricity is considered according to the above mentioned formula, the same results were obtained whether the location of appearance or disappearance of the stimulus was determined.

One of the principal findings of this study was that the eccentric visibility of black stimuli was less than that of white stimuli of equivalent size. There are several possible explanations for this phenomenon. Firstly, light scatter from the bright surface into the adjacent dark surface on the campimeter may have caused geometrical change in the perceived sizes of the stimuli, the apparent size of the black stimulus being smaller compared with the white stimulus. Secondly, the light stimuli moved through relatively dark adapted, and correspondingly more sensitive parts of the visual field than the dark stimuli, which were presented to bleached parts of the retina. This explanation is implausible in view of the fact that the black stimulus was less visible than the white one even with a grey background. Furthermore, the phenomenon was observed under photopic conditions.

An alternative explanation for the reduced visibility of the dark stimulus may be the fact that it depended mainly on off centre ganglion cells, which in the central field are less abundant than on centre ganglion cells (that is, in a ratio of approximately 1-2:3). Similar psychophysical differences between these two parallel visual pathways have previously been reported for foveal vision with regard to target detection rates and reaction times. ${ }^{23}{ }^{24}$ In addition, electrophysiological differences between these two channels have been observed..$^{25}$ We are unaware of a previous report of perimetric asymmetries between on and off pathways. If the black perimetric stimulus is indeed testing the off pathway, it may prove to be superior to conventional luminous stimuli. Glaucoma, for example, is believed to affect the peripheral contrast sensitivity, which is the functional result of the balance between on and off pathways. ${ }^{1927} 28$

The second finding of this study was that the visibility of the black stimulus was perceived by the subjects as being more constant under varying lighting conditions than that of the white stimulus. This difference was observed even when the same grey background was used for both stimuli. One reason for this finding may be that at the two different levels of illumination the differences in the amount of the light reflected by the black and white stimuli surface were not constant. However, both type of stimuli maintained the same equal and opposite sign luminance (Weber's) contrast at each illuminance level (Fig 1). Therefore an alternative explanation is that on and off systems may behave differently with changing levels of ambient illumination. The practical significance of this difference is that standardisation of the ambient illumination may be less important when perimetry is performed with dark stimuli on a bright background than vice versa. This would render the dark perimetric stimulus superior to the conventional
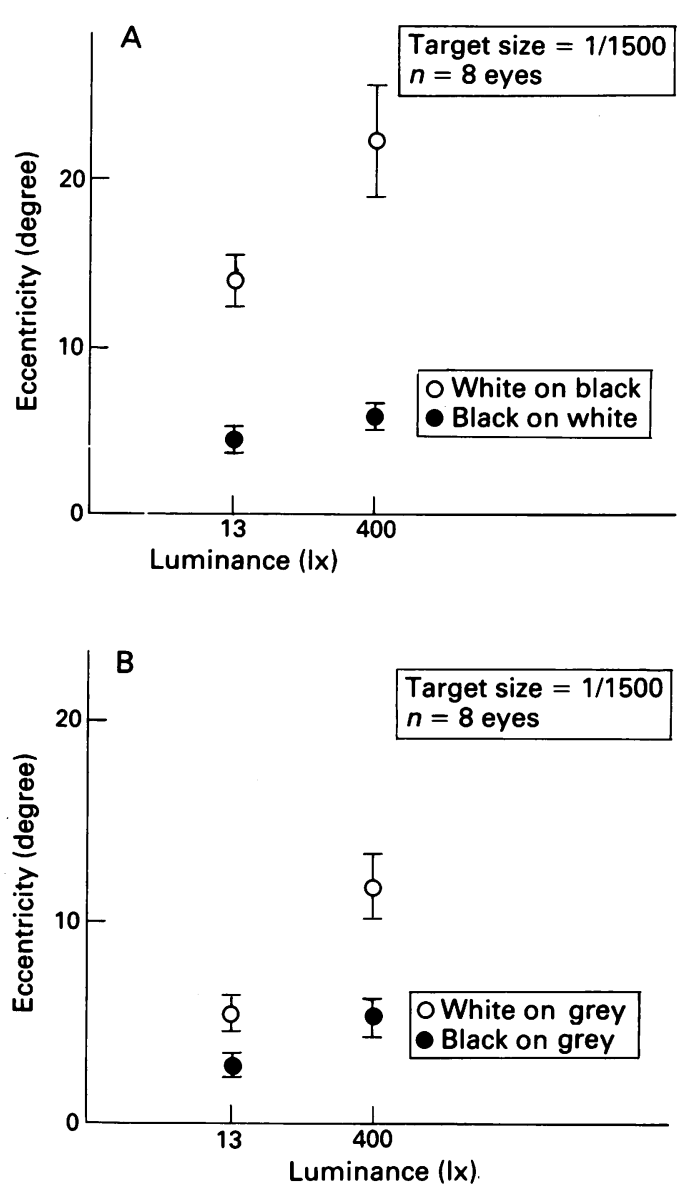

Figure $3 A$ and $B$ The effect of variation in surrounding illumination on the perimetric visibility of equal dark and bright stimuli on tangent screen (mean with SD). The dark stimulus visibility displayed significantly less change with changing level of illumination both on opposite and grey backgrounds. (The illuminance difference is 1.5 log.) 
light stimulus when visual field examination is performed in the community and in other situations where it is difficult to maintain standardised and constant lighting conditions.

In conclusion, this study has shown that the black perimetric stimulus is less visible and more stable under variable lighting conditions than the light stimulus. Whether such differences result from the differential stimulation of the on and off visual pathways merits further study.

Dr David Keating helped us on the statistical evaluation of the results. We were financially supported by International Glaucoma Association.

1 Reed H, Drance MS. The essentials of perimetry, static and kinetic. 2nd ed. London: Oxford University Press, 1972.

2 Harrington DO, Drake MV. The visual fields: text and atlas of clinical perimetry. 6th ed. St Louis: Mosby, 1990

3 Anderson DR. Perimetry with and without automation. 2nd ed. St Louis: Mosby, 1987.

4 Wagner HG, MacNichol EF, Wolbarsht ML. Functional basis for on-center and off-center receptive fields in the retina f Opt Soc Am 1963; 53: 66-70.

5 Derrington AM, Lennie P. Spatial and temporal contrast sensitivities of neurones in lateral geniculate nucleus of macaque. F Physiol (Lond) 1984; 357: 219-40.

6 Perry VH, Cowey A. The morphological correlates of X-and $Y$-like retinal ganglion cells in the retina of monkeys. Exp Brain Res 1981; 43: 226-8.

7 de Monesterio FM, Gouras P. Functional properties of ganglion cells of the rhesus monkey retina. $\mathcal{F}$ Physiol (Lond) 1975; 251: 167-95.

8 Wassle $\mathbf{H}$. Sampling of visual space by retinal ganglion cells. Invest Ophthalmol Vis Sci 1988; 29 (suppl): 117.

9 Bishop PO. Processing of visual information within the retinostriate system. In: Darian-Smith I, ed Handbook of physiology. Bethesda: American Physiological Society, 1984.

10 Kennard C, Rose FC. Physiological aspects of clinical neuro ophthalmology. London: Chapman and Hall, 1988.

11 Drance SM, Anderson DR. Automatic perimetry in glaucoma. Orlando: Grune and Stratton, 1985.

12 Perry VH, Silveria LCL. Functional lamination in the ganglion cell layer of the macaque's retina. Neuroscience 1988; 25: 217-24.
13 Quigley HA, Dunkelberger GR, Green WR. Retinal ganglion cell atrophy correlated with automated perimetry in human eyes with glaucoma. Am 7 Ophthalmol 1989; 107: 453-64. 14 Saito T. Physiological and morphological differences between on- and off-center bipolar cells in the vertebrate retina. Vision Res 1987; 27: 135-42.

15 Schiller PH, Sandell JH, Maunsell JHR. Functions of the on and off channels of the visual system. Nature 1986; 322: $824-5$.

16 Nelson R, Famiglietti EV, Kolb H. Intracellular staining reveals different levels of stratification for on- and off-center ganglion cells in cat retina. 7 Neurophysiol 1978; 41: 472-83.

17 Peichl L, Wassle H. Morphological identification of on- and off-centre brisk transient cells in the cat retina. Proc $R$ Soc Lond Biol 1981; 212: 139-56.

18 Kageyama GH, Wong-Riley MTT. The histochemical localisation of cytochrome-oxidase in the retina and lateral geniculate nucleus of the ferret, cat, and monkey, with particular reference to retinal mosaics and on/off center visual channels. F Neurosci 1984; 4 : 2445-59.

19 Liu S, Wong-Riley M. Quantitative light and electron microscopic analysis of cytochrome-oxidase distribution in neurons of the lateral geniculate nucleus of the adult monkey. Vis Neurosci 1990; 4: 269-87.

20 Wassle H, Peichl L, Boycott BB. A spatial analysis of on- and off-ganglion cells in the cat retina. Vision Res 1983; 23: 115160.

21 Gouras $\mathrm{P}$, Zrenner E. Colour coding in primate retina. Vision Res 1981; 21: 1591-8.

22 Damato BE. Oculo-kinetic perimetiy: a simple visual field test for use in the community. BrF Ophthalmol 1985; 69: 927-31.

23 White TW, Irwin GE, Williams M. Asymmetry in the brightness and darkness Broca-Sulzer effects. Vision Res 1980; 20: 723-6.

24 Rea MS, Oullette MJ. Visual performance using reaction times. Lighting Res Technol 1988; 20: 139-53.

25 Zemon V, Gordon J, Welch J. Asymmetries in on and off visual pathways of humans revealed using contrast-evoked cortical potentials. Vis Neurosci 1988; 1: 145-50.

26 Bowen RW, Pakorny J, Smith VC. Sawtooth contrast sensitivity: decrements have the edge. Vision Res 1989; 29 . 1501-9.

27 Falco-Reis F, O'Donaghue E, Buceti R, Hitchings RA, Arden GB. Peripheral contrast sensitivity in glaucoma and ocular hypertension. Br f Ophthalmol 1990; 74: 712-6.

28 Howe JW, Mitchell KW. Electrophysiologically determined contrast sensitivity in patients with ocular hypertension and
chronic glaucoma. Presented at the XXIX Symposium of the chronic glaucoma. Presented at the XXIX Symposium of the Vision, 2-6 July, 1991, Oxford, England. 